\title{
The Effect of Histidine-tryptophan-ketoglutarate Solution and University of Wisconsin Solution: An Analysis of the Eurotransplant Registry
}

Jacob D. de Boer, MD, ${ }^{1,2}$ Agita Strelniece, ${ }^{3}$ Marieke van Rosmalen, MD, ${ }^{1}$ Erwin de Vries, ${ }^{1}$ Dirk Ysebaert, MD, PhD, ${ }^{4}$ Markus Guba, MD, PhD, ${ }^{5}$ Andries Erik Braat, MD, PhD, ${ }^{2}$ and Undine Samuel, MD, PhD ${ }^{1}$

Background. Both University of Wisconsin (UW) and histidine-tryptophan-ketoglutarate (HTK) solutions are currently used in the Eurotransplant region for preservation of liver allografts. Previous studies on their effect have led to a lot of discussion. This study aims to compare the effect of HTK and UW on graft survival. Methods. First liver transplantations in recipients 18 years or older from January 1, 2007, until December 31, 2016, were included. Graft survival was compared for livers preserved with HTK and UW at 30 days, 1, 3, and 5 years. Multivariable analysis of risk factors was performed and outcome was adjusted for important confounders. Results. Of all 10628 first liver transplantations, 8176 (77\%) and 2452 (23\%) were performed with livers preserved with HTK and UW, respectively. Kaplan-Meier curves showed significant differences in graft survival between HTK and UW at 30 days (89\% vs 93\%, $P=<0.001)$, 1 year (75\% vs $82 \%, P=<0.001), 3$ years $(67 \%$ vs $72 \%, P<0.001)$, and at 5 years $(60 \%$ vs $67 \%, P<0.001)$. No significant differences in outcome were observed in separate analyses of Germany or non-German countries. In multivariable analysis, UW was associated with a decreased risk of graft loss at 30 days (HR $0.772, P=0.002)$ and at 1 year (0.847 (0.757-0.947). When adjusted for risk factors, no differences in long term outcome could be detected. Conclusions. Because the use of preservation fluids is clustered geographically, differences in outcome by preservation fluids are strongly affected by regional differences in donor and recipient characteristics. When adjusted for risk factors, no differences in graft survival exist between transplantations performed with livers preserved with either HTK or UW.

(Transplantation 2018;102: 1870-1877)

\begin{abstract}
schemic injury sustained during organ preservation influences posttransplantation outcomes in an important way. Throughout the process of organ preservation, preservation fluids are used. In the donor, the liver is perfused with cold preservation fluid after cross-clamping of the aorta. It is then packed in a sterile bag filled with this same fluid in a box with ice after hepatecomy. ${ }^{1}$ In the transplant hospital, the organ is perfused before transplantation using the same preservation fluid. Almost all livers within Eurotransplant (ET) are preserved by this "cold storage." Other preservation techniques,
\end{abstract}

\footnotetext{
Received 22 February 2018. Revision received 18 May 2018.

Accepted 29 May 2018.

1 Eurotransplant International Foundation, Leiden, The Netherlands.

2 Department of Surgery, Leiden University Medical Center, Leiden, The Netherlands.

${ }^{3}$ Biostatistical Department, Eurotransplant International Foundation, Leiden, The Netherlands.

${ }^{4}$ Department of Hepatobiliary, Transplantation and Endocrine Surgery, Antwerp University Hospital, University of Antwerp, Belgium.

${ }^{5}$ Department of General, Visceral, Transplantation, Vascular and Thoracic Surgery,
} Hospital of the University of Munich, Munich, Germany. such as machine perfusion, are currently only performed in an experimental way.

Several preservation fluids are used within the ET region although most countries use either University of Wisconsin (UW) solution or histidine-tryptophan-ketoglutarate (HTK) solution. $^{2}$ The choice of preservation fluid is thought to be important for outcome and a difference in effect on outcome has often been studied. First, studies on the topic could not detect significant differences in short- and long-term patient and graft survivals ${ }^{2-7}$ (Table 1). This might have been a result

Jd.B. and U.S. participated in the study concept and design. Mv.R. and E.V. participated in the acquisition of data. Jd.B., A.S. participated in the statistical analysis. Jd.B., A.S., E.V., A.B. participated in the analysis and interpretation of data. Jd.B., U.S., Mv.R., A.B. participated in the drafting of the article. M.G., A.B., U.S., D.Y., Mv.R., E.V. participated in the critical revision of the article. A.S., U.S. participated in the study supervision.

On behalf of the Eurotransplant Liver and Intestine Advisory Committee (ELIAC) and Organ Procurement Process Chain Committee (OPCC).

Correspondence: J.D. de Boer, MD, Eurotransplant International Foundation, Leiden, The Netherlands. (jacob.deboer@eurotransplant.org).

Supplemental digital content (SDC) is available for this article. Direct URL citations appear in the printed text, and links to the digital files are provided in the HTML text of this article on the journal's Web site (www.transplantjournal.com).

Copyright (C) 2018 Wolters Kluwer Health, Inc. All rights reserved.

ISSN: 0041-1337/18/10211-1870

DOl: $10.1097 /$ TP.0000000000002406 
TABLE 1.

Studies on the use and effect of perfusion fluids in deceased donor liver transplantation



of the frequent single-center design and low numbers of included transplantations. A larger study by Stewart et $\mathrm{al}^{10}$ showed HTK to be associated with a higher risk of early graft loss (<30 days) as compared with UW in the United Network for Organ Sharing (UNOS) database. It contributed to a gradual change to UW although some centers prefer HTK for the lower viscosity and lower costs.

More recent studies of Kaltenborn et $\mathrm{al}^{9}$ and Adam et $\mathrm{al}^{8}$ presented conflicting results on the issue. Kaltenborn showed only minimal differences between HTK and UW, whereas Adam et al found HTK to be associated with a significant increased risk of long-term graft loss (at least up to 5 years) as compared with UW in the European Liver Transplant Registry (ELTR). ${ }^{8}$ Several remarks and concerns with the design of the study and its conclusions were placed by Nashan et al. ${ }^{11}$ Most important concerns were with including living donation, insufficient risk adjustment and the overrepresentation of German livers in the HTK group. Germany uses HTK exclusively and it has a model for end-stage liver disease (MELD)-based allocation combined with one of the lowest donor rates of Europe. ${ }^{12}$ The difference in long-term outcome that was attributed to HTK in this study might rather reflect inferior outcomes in general in Germany. In response, Adam et al published an analysis without living donors and German centers and more recently, an analysis based on propensity score matching. ${ }^{8,13}$ This analysis matched patients on ABO compatibility; recipient ischemic time of 6 hours or longer; sex; study period (2003-2007 vs 2008-2012); recipient age, 60 years or older, donor age, 55 years or older; whole liver; urgency of transplantation; hepatocellular carcinoma; recipient HIV status; and centers performing more than 10 liver transplantations from living donors. Although an association between HTK and graft loss could be seen, we believe that interregional differences in donor, transplant, and recipient characteristics were insufficiently taken into account.

This study aims to evaluate the effect of HTK and UW on short- and long-term outcomes after liver transplantation in the Eurotransplant region, with adequate adjustment for (regional) differences in donor, transplant, and recipient factors.

\section{PATIENTS AND METHODS}

\section{Data Selection}

All first transplantations from deceased donor livers performed in adult recipients ( $\geq 18$ years) from January 1, 2007, until December 31, 2016, were included. Transplantations with livers from donors after circulatory death (DCD) ( $\mathrm{n}=771)$, split allografts $(\mathrm{n}=380)$ and allografts from donors outside of Eurotransplant were excluded. When information on the used preservation fluids was missing $(\mathrm{n}=160)$ or when preserved with other preservation fluids than HTK or UW fluid (Celsior $\mathrm{n}=18$, Eurocollins = 1, IGL-1 $n=79$ and other $n=216$ ) transplantations were also excluded as well as transplantations performed in patients with a high-urgency status $(n=888)$, with a combination other than liver/kidney and transplantations performed in Göttingen. ${ }^{14}$ Transplantations were categorized in either HTK or UW according to the preservation fluid that was used during procurement and subsequent transport. 
Follow-up data were obtained from the Eurotransplant Network Information System and ET Liver Registry up to September 2017. All data were anonymized for transplant center and patient related data with exception of country. The study protocol was approved by the Eurotransplant Liver Intestine Advisory Committee, and no ethical statement was required according to European guidelines and Dutch law.

\section{Data Analysis}

Laboratory values were converted to standardized units and in case of missing values $<2 \%$, median values were used; gamma-glutamyl-transpeptidase (GGT) $38 \mathrm{U} / \mathrm{L}$ $(1.8 \%)$ and recipient body mass index (BMI) $25.8(0 \%)$. The Eurotransplant-Donor Risk Index (ET-DRI) ${ }^{15}$ was calculated for all transplanted livers and the simplified recipient risk index $(\mathrm{sRRI})^{16}$ was calculated for all recipients based on most recent laboratory MELD score before transplantation. With the ET-DRI and sRRI the donor-recipient model (DRM) was calculated for all transplantations. ${ }^{17}$ Serum creatinin value was set at $4 \mathrm{mg} / \mathrm{d}$ therapy according to ET guidelines for patients receiving renal replacement, MELD score was rounded to the nearest whole value (range, 6-40). Donor hepatitis $C$ antibody (HCVAb), donor hepatitis B core antibody ( $\mathrm{HBcAb}$ ), recipient $\mathrm{HCVAb}$, dialysis of the recipient before transplantation and a history of diabetes in the donor were considered negative if not tested or missing. Rescue allocation is a center-oriented allocation after patient-oriented allocation and is started for short allocation time or medical reasons. Clinical characteristics were summarized by median and $25 \%$ and $75 \%$ interquartile ranges (IQR) and number and percentage (N/\%) for respectively continuous and categorical variables. Numerical and categorical factors between groups were compared using Kruskall-Wallis and $\chi^{2}$ tests.

\section{Outcome Measures}

Primary outcomes used in the analyses were 30 days, 1-, 3-, and 5-year non-death-censored graft survival. Secondary outcomes were 30 days, 1-, 3-, and 5-year patient survival (PS). Graft survival was defined as the period between date of transplantation and date of retransplantation or patient death. Patient survival was defined as the period between date of transplantation and date of patient death. Outcome was analyzed by Kaplan Meier analysis and log-rank tests when stratified by preservation fluid category (HTK, UW). Results were also stratified for transplantation region and preservation fluid (Germany+HTK, Germany+UW and non-Germany+HTK, non-Germany+UW).

\section{Risk Factors}

To identify risk factors associated with graft survival, multivariable analysis was performed in a Cox regression analysis (backward selection) for all transplantations and included factors described to be associated with graft survival. ${ }^{15,17-19}$ These factors included donor age, cause of death, sex, BMI, latest GGT, HBcAb, HCVAb, history of diabetes, Recipient age, sex, BMI, laboratory MELD score at transplantation, etiology of primary liver disease, liver/kidney combination, dialysis before transplantation, total ischemic time, rescue allocation, allocation region (local, regional, extraregional), and year of transplantation (continuous). Graft survival was then adjusted for all risk factors associated with 5-year graft survival in Germany, non-German countries and all transplantations. A potential effect of preservation fluids in
HCC patients or in livers with longer cold ischemic times was described in literature. ${ }^{8}$ This potential relation was analyzed with Kaplan-Meier analysis and in a Cox-regression analysis when adjusted for risk factors.

For all analyses a Wald $P$ value less than 0.05 was considered significant. Survival analyses were performed using Kaplan-Meier survival models and multivariable analyses were performed using Cox regression models. All analyses were performed with SPSS (version 24.0).

\section{RESULTS}

Within the study period, 10628 first liver transplantations were included. Median donor age of all transplantations was 55 years old (IQR, 45-67) and median donor BMI 26 (IQR, 24-28). Cerebrovascular accident was the most frequent cause of death $(62 \%)$ followed by trauma $(20 \%)$. Near half of donors was allocated extraregionally $(46 \%)$ and median ET-DRI was 1.84. Most recipients were male $(70 \%)$ and had a median age 56 years old and median BMI of 25 . Transplanted recipients had a median laboratory MELD score of 16 and a median match MELD score of 24. Alcoholic disease was most frequent primary diagnosis $(27 \%)$ followed by malignant disease $(25 \%)$ and other cirrhosis $(14 \%)$. The majority of transplantations was performed in Germany $(62 \%)$ followed by Belgium (12\%) and Austria (10\%). Median sRRI was 1.86 and median DRM was 2.77.

\section{Preservation Fluid Category}

Of all transplantations, 8176 (77\%) and 2452 (23\%) were performed with livers preserved with HTK and UW, respectively. The relative use of UW decreased from 36\% in 2007 to $18 \%$ in 2016 , whereas the use of HTK increased from $64 \%$ to $82 \%$ (Figure 1 ). Within donor countries strong preference for either HTK or UW during procurement was seen. HTK is preferred in Hungary $(100 \%)$, Germany $(98 \%)$, Slovenia (97\%) and Austria (84\%), whereas UW is preferred in The Netherlands (98\%), Croatia (83\%), Belgium $(73 \%)$ and, with very small numbers, Luxembourg (100\%).

Median donor age and BMI were significantly higher in the HTK group as compared with the UW group ( 56 years vs 55 years, $P<0.001$ ) and (26 years vs 25 years, $P<0.001$ ), respectively. Cause of death of the donor was significantly



FIGURE 1. The use of HTK and UW in the Eurotransplant region. 
TABLE 2.

Donor and recipient characteristics per perfusion fluid, $n=10826$

\begin{tabular}{|c|c|c|c|}
\hline & $\begin{array}{l}\text { HTK Bretschneider } \\
\quad(\mathrm{n}=\mathbf{8 1 7 6})\end{array}$ & $\begin{array}{c}\text { UW } \\
(n=2452)\end{array}$ & $\begin{array}{l}\text { HTK } \\
\text { vs UW }\end{array}$ \\
\hline & $\begin{array}{c}\text { (n (\%)/ } \\
\text { Median (25\%/ } \\
75 \% \text { percentile) }\end{array}$ & $\begin{array}{c}(\mathrm{n}(\%) / \\
\text { Median (25\%/ } \\
75 \% \text { percentile) }\end{array}$ & $P$ \\
\hline \multicolumn{4}{|l|}{ Donor factors } \\
\hline Donor age, y & $56(45-67)$ & $55(43-65)$ & $<0.001$ \\
\hline Height, cm & $174(165-180)$ & $174(167-180)$ & 0.097 \\
\hline Weight, kg & 80 (70-90) & $76(68-85)$ & $<0.001$ \\
\hline $\mathrm{BMI}$ & $26(24-28)$ & $25(23-28)$ & $<0.001$ \\
\hline Last GGT, U/L & 43 (22-99) & 31 (17-62) & $<0.001$ \\
\hline Sex (male) & $4445(54)$ & $1366(56)$ & 0.241 \\
\hline Cause of death & & & $<0.001$ \\
\hline Anoxia & $1020(13)$ & $82(3)$ & \\
\hline Circulational & $113(1)$ & $158(6)$ & \\
\hline CNS tumor & $44(1)$ & $19(1)$ & \\
\hline CVA/stroke & $5129(63)$ & $1484(61)$ & \\
\hline Trauma & $1426(17)$ & $648(26)$ & \\
\hline Other & $443(5)$ & $61(3)$ & \\
\hline Diabetes, y & $816(10)$ & $173(7)$ & $<0.001$ \\
\hline \multicolumn{4}{|l|}{ Transplant factor } \\
\hline Total ischemic time, $\mathrm{h}$ & $8.6(6.3-11.0)$ & $7.3(5.0-9.6)$ & $<0.001$ \\
\hline Allocation region & & & $<0.001$ \\
\hline Local & $1980(24)$ & $1004(41)$ & \\
\hline Regional & $1902(23)$ & $892(36)$ & \\
\hline Extraregional & $4294(53)$ & $556(23)$ & \\
\hline Rescue (yes) & $2613(32)$ & 389 (16) & $<0.001$ \\
\hline Country & & & $<0.001$ \\
\hline Germany & $6147(75)$ & $463(19)$ & \\
\hline Hungary & $221(3)$ & $11(0)$ & \\
\hline The Netherlands & $124(2)$ & 465 (19) & \\
\hline Belgium & $476(6)$ & 752 (31) & \\
\hline Croatia & $196(2)$ & $593(24)$ & \\
\hline Slovenia & $149(2)$ & $9(0)$ & \\
\hline Austria & $863(11)$ & $159(7)$ & \\
\hline ET-DRl & $1.90(1.59-2.24)$ & 1.66 (1.40-1.92) & $<0.001$ \\
\hline \multicolumn{4}{|l|}{ Recipient factor } \\
\hline Age, y & 56 (49-62) & 57 (49-62) & 0.093 \\
\hline Height, cm & $174(168-180)$ & $173(167-180)$ & 0.003 \\
\hline Weight, kg & $80(69-90)$ & $78(68-90)$ & 0.019 \\
\hline BMl & $26(23-29)$ & $26(23-29)$ & 0.390 \\
\hline Laboratory MELD & $16(11-27)$ & $16(11-23)$ & 0.001 \\
\hline Match MELD & $25(16-31)$ & $22(17-27)$ & $<0.001$ \\
\hline Exceptional MELD (yes) & $2753(34)$ & 790 (32) & 0.181 \\
\hline Sex (male) & $5759(70)$ & 1696 (69) & 0.228 \\
\hline Dialysis pretransplant & 1002 (12) & $157(6)$ & $<0.001$ \\
\hline Primary diagnosis & & & $<0.001$ \\
\hline Metabolic & $264(3)$ & $91(4)$ & \\
\hline Acute & $158(7)$ & $28(1)$ & \\
\hline Cholestatic & $906(10)$ & $267(11)$ & \\
\hline Alcoholic & $2112(24)$ & 716 (29) & \\
\hline Malignant & $2060(24)$ & $628(26)$ & \\
\hline HBV & $316(4)$ & $94(4)$ & \\
\hline $\mathrm{HCV}$ & $867(10)$ & $211(9)$ & \\
\hline Other cirrhosis & 1146 (13) & 295 (12) & \\
\hline Other & $347(5)$ & $122(5)$ & \\
\hline
\end{tabular}

TABLE 2. (Continued)

\begin{tabular}{llcc}
\hline & $\begin{array}{c}\text { HTK Bretschneider } \\
(\mathbf{n = 8 1 7 6 )}\end{array}$ & $\begin{array}{c}\text { UW } \\
(\mathbf{n}=\mathbf{2 4 5 2})\end{array}$ & $\begin{array}{c}\text { HTK } \\
\text { vs UW }\end{array}$ \\
\hline $\begin{array}{l}\text { LabMELD category } \\
<15\end{array}$ & $3515(43)$ & $1040(42)$ & $<0.001$ \\
$15-25$ & $2446(30)$ & $930(38)$ & \\
$25-35$ & $1136(14)$ & $329(13)$ & \\
$35+$ & $1079(13)$ & $153(6)$ & \\
SRRI & $1.87(1.58-2.23)$ & $1.86(1.58-2.17)$ & $<0.001$ \\
DRM & $2.85(2.31-3.51)$ & $2.56(2.09-3.08)$ & $<0.001$ \\
\hline
\end{tabular}

different between both groups $(P<0.001)$; less trauma $(17 \%$ vs $26 \%)$ and more often anoxia $(13 \%$ vs $3 \%)$ were registered as cause death in the HTK group. Total ischemic times were longer in the HTK group in comparison to the UW group (8.6 hours vs 7.3 hours) and HTK livers were more often accepted in rescue allocation $(32 \%$ vs $16 \%$, $P<0.001)$. The median ET-DRI was significantly higher in the HTK group $(1.90$ vs $1.66, P<0.001)$.

Recipient age and BMI were not different in both the UW and HTK group with a median of 56 years old $(P=0.093)$ and BMI of $26(P=0.390)$, respectively. Although both groups had a similar median laboratory MELD score, the distribution was not equal $(P<0.001)$. As compared with the UW group, the HTK group has a higher proportion of transplanted MELD 25-35 (14\% vs 13\%) and MELD 35+ recipients $(13 \%$ vs $6 \%)$. Also, the match MELD did vary between HTK and UW (25 vs $22, P<0.001$ ). Median sRRI showed only minor differences, whereas the DRM was significantly higher in the HTK group 2.85 versus $2.56(P<0.001)$, data shown in Table 2.

\section{Outcome}

For all transplantations, graft survival at 30 days, 1,3 and 5 years was $90 \%, 77 \%, 68 \%$, and $62 \%$, respectively. Graft survival was significantly better in the UW group as compared with HTK at 30 days $(93 \%$ vs $89 \%, P=<0.001)$, 1 year $(82 \%$ vs $75 \%, P=<0.001)$, 3 years $(72 \%$ vs $67 \%$, $P<0.001)$, and at 5 years $(67 \%$ vs $60 \%, P<0.001)$, as shown in Figure 2A. Similar differences were found in PS; transplantations with UW-preserved livers showed better PS as compared with HTK at 30 days $(95 \%$ vs $93 \%$, $P=<0.001), 1$ year $(86 \%$ vs $79 \%, P=<0.001), 3$ years ( $78 \%$ vs $71 \%, P<0.001)$, and at 5 years $(72 \%$ vs $65 \%$, $P=<0.001)$, as shown in Figure 2B.

Within Germany, 6174 transplantations were performed with HTK and 463 with UW. In non-German countries, 2029 and 1989 transplantations were performed with HTK- and UW-preserved livers, respectively. Outcome stratified for transplantation region (Germany/non-Germany) and preservation fluid (HTK/UW) showed significantly lower overall graft survival in Germany. Within both regions, a trend for a slightly higher graft survival on short term was seen for UW-preserved livers as compared with HTK livers. On long-term, HTK livers showed a trend toward better graft survival. This was observed in Germany at 30 days (HTK $87 \%$ vs UW $88 \%$ ), 1 year (HTK, $72 \%$ vs UW, $73 \%$ ), 3 years (HTK 64\% vs UW 64\%), and at 5 years (HTK, 57\% vs UW, 56\%). In non-Germany this was also observed at 30 days (HTK 93\% vs $94 \%$ ), 1 year (HTK, $83 \%$ vs 
A

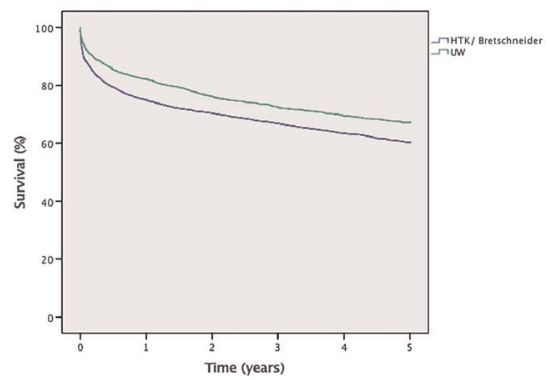

B

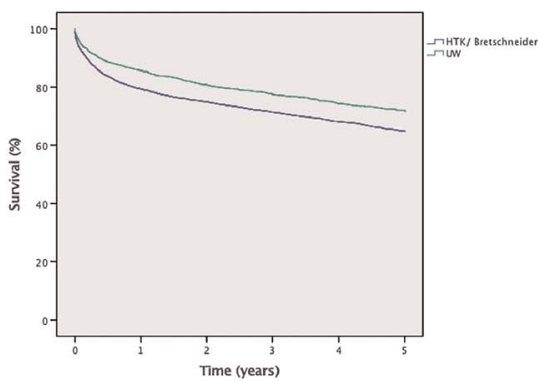

FIGURE 2. Kaplan-Meier survival analysis by preservation fluid $(n=10628)$. A, Graft survival. B, Patient survival.

$84 \%$ ), 3 years (HTK, $76 \%$ vs UW, $74 \%$ ) and at 5 years $(70 \%$ vs $70 \%$ ) (data shown in Figure 3). Differences in outcome within both regions were not statistically significant at any time point.

\section{Risk Factors}

In multivariable analysis, donor age, total ischemic time, donor last GGT, a history of diabetes in the donor, allocation region, rescue, recipient age, sex, etiology of liver disease, dialysis before transplantation, laboratory MELD score and year of transplantation were associated with 5-year graft survival. An association between outcome and preservation fluids could only be detected on short-term. UW was associated with a decreased risk of graft loss at 30 days (hazard ratio [HR], 0.762; 95\% confidence interval [CI], $0.643-0.902 ; P=0.002)$ and at 1 year $(\mathrm{HR}, 0.835 ; 95 \%$ CI, 0.746-0.0.934; $P=0.002$ ), data are shown in Table 3. When adjusted for all risk factors associated with 5 -years graft survival, no difference could be detected between both preservation fluids in transplantations performed in Germany $(P=0.572)$ (Figure $4 \mathrm{~A})$ or non-Germany $(P=0.522)$ (Figure 4B). In all transplantations, also no difference in longterm outcome could be shown (data are shown in Figure 4C).

\section{Risk Groups}

Of all transplantations, 3527 (33\%) of patients had a registered HCC. Patients with HCC had lower graft survival when transplanted with a liver preserved with HTK $(\mathrm{n}=2747)$ as compared with livers preserved with UW $(\mathrm{n}=780)$ at 30 days $(90 \%$ vs $93 \%, P=0.013)$ and at 1 year ( $77 \%$ vs $81 \%, P=0.006$ ). When adjusted for other risk factors, a potential effect of HTK or UW in HCC patients was not observed at 30 days $(P=0.557)$ or at 1 year $(P=0.424)$.

When transplantations were stratified according to the ELTR total ischemic times categories, 3 groups were identified; livers transplanted with $<=6$ hours $(\mathrm{n}=2700), 6-12$ hours $(\mathrm{n}=6231)$ and $>=12$ hours $(\mathrm{n}=1697)$ of cold ischemic time. Only in transplantations performed with livers with 6 to 12 hours of cold ischemic time a statistically significant difference between HTK and UW could be observed $(60 \%$ vs $69 \%$, $P<0.001$ ) (data are shown in Figure S1a-c, SDC, http://links. lww.com/TP/B622). When adjusted for other risk factors, or when analyzed per region (Germany vs non-Germany) this potential negative impact of HTK in livers with longer cold ischemic times was not observed (data are shown in Figure S2-3a, b, c, SDC, http:/links.lww.com/TP/B622).

\section{DISCUSSION}

This study shows that HTK is used in the majority of organ transplantations within Eurotransplant. The use of HTK is increasing, in contrast to UW. Overall graft survival is lower for livers preserved with HTK, but these results are strongly affected by regional differences in donor, recipient and transplant characteristics. When adjusted for these risk factors, no difference between HTK and UW could be observed.

The issue of preservation fluids remains an important point of discussion in liver transplantation. Although evidence is still considered nonconclusive, different preservation fluids are currently used. This study shows that although UW is internationally considered the golden standard, the relative use of UW within ET is decreasing, whereas the use of HTK is increasing. To compare the effect of both preservation fluids, we have tried to ensure a homogenous study population. We have excluded all pediatric recipients, those receiving living related livers, livers from DCD donors, split livers and transplantations in high-urgent patients. Even with these strict inclusion criteria, this study includes a sufficiently high number of transplantations to detect minor differences in outcome and to perform an adequate multivariable analysis. The unfavorable characteristics of the group of livers preserved with HTK are likely to have contributed to the inferior graft survival and PS. We have therefore separated our analysis per region, and have adjusted outcome for risk factors to interpret the differences in graft survival and PS. The high completeness for important data like total ischemic times and MELD

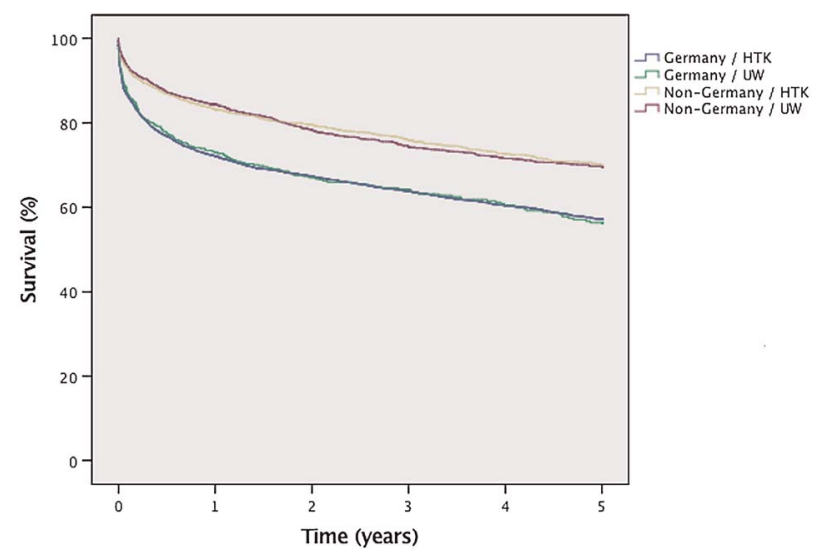

FIGURE 3. Kaplan Meier survival analysis of graft survival by preservation fluid and transplant region (Germany vs non-Germany), $(n=10628)$. 
TABLE 3.

Multivariable analysis of factors associated with graft survival

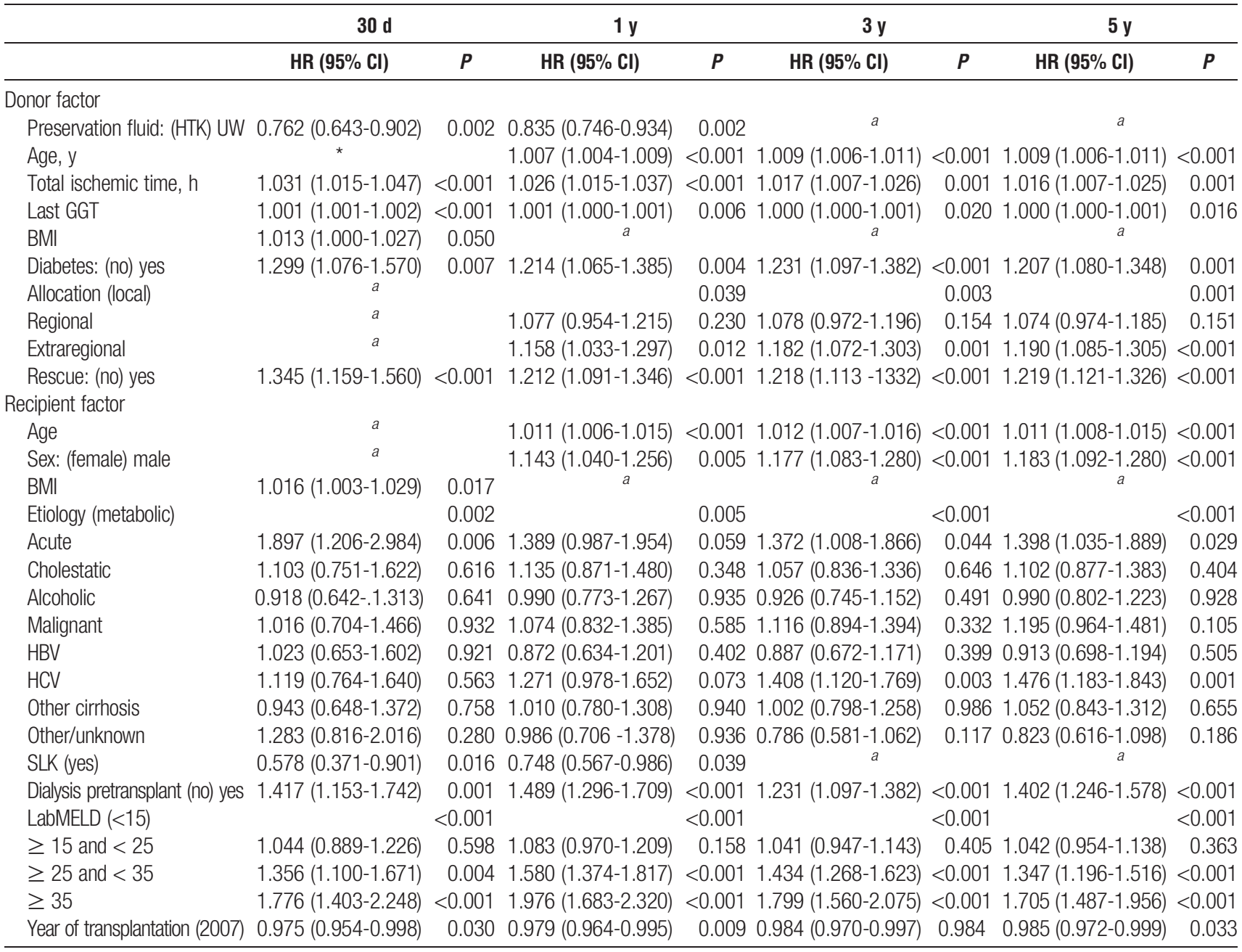

${ }^{a}$ No statistical significance and not in the equation.

The following factors were not statistically significantly associated with outcome at the measured time points: donor sex, cause of death, HBCAb, HCVAb, recipient sex, HCVAb.

score add to the reliability of our findings. Although performed with care, risk adjustment may still not be sufficient as is inherent to the retrospective design. We considered graft survival as primary outcome and did not have information on biliary complications or early bile production. This is a potential limitation, because some studies found suggestions for more posttransplantation bile production and less biliary complications in livers that were preserved with HTK. ${ }^{20}$ However, biliary complications will likely also affect graftsurvival in the long run.

The presented results of inferior unadjusted graft survival between HTK and UW are in line with the previously published study by the ELTR. ${ }^{8}$ The ELTR study attributed this inferior long-term outcome to the use of HTK. Interesting, because the risk of HTK on graft loss was one of the lowest of all risk factors and only just statistically significant (risk ratio $1.1, P=0.02$ ) in over 34500 transplantations. ${ }^{8}$ Based on our findings, differences in long-term outcome in particular, are more likely to reflect differences in donor, recipient and transplant risks than an effect of the preservation fluid itself. When these differences are adequately taken into account no statistically significant difference could be detected between HTK and UW. This finding is in accordance to other studies that could not show any significant differences between HTK and $\mathrm{UW}^{2-7}$ Although this could be a result of an inadequate power due to small numbers, also Kaltenborn et $\mathrm{al}^{9}$ neither have shown a difference in risk between both fluids despite a sizeable data set (summary in Table 1). A slightly better short term graft survival in livers preserved with UW, as reported by Stewart et $\mathrm{al},{ }^{10}$ may be present according to the risk adjusted survival in non-German countries (Figure 3B).

Some studies have also described a more pronounced effect of preservation fluids in several subgroups. This would affect livers from DCD donors, ${ }^{10}$ livers with total ischemic times longer than 12 hours, ${ }^{8}$ patients with a $\mathrm{HCC}^{8}$ and split liver allografts. ${ }^{8}$ A potential difference in DCD donors and split procedures could not be analyzed because these were excluded in this study. Differences in the other mentioned subgroups (categorical total ischemic time groups, HCC recipients) were not confirmed in this study or did not persist when adjusted for other risk factors.

To correctly interpret differences in outcome between several preservation fluids, the hypothesized causative pathway is important. The mechanism through which HTK would 
A

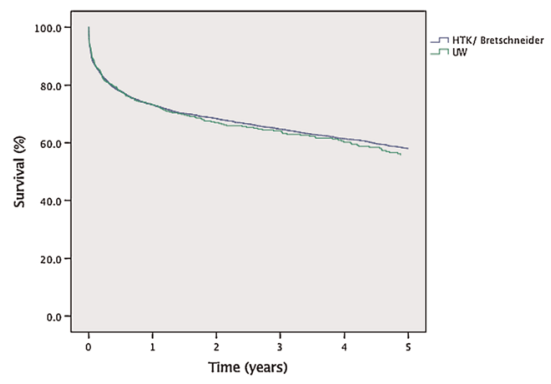

B



C

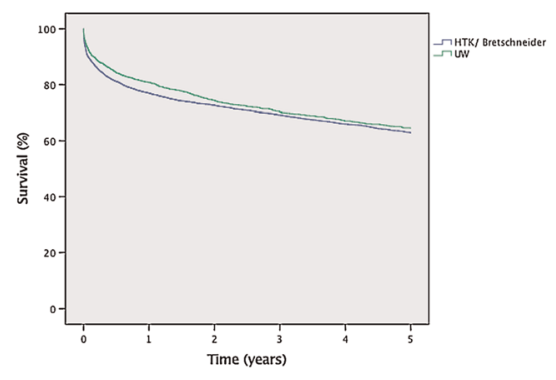

FIGURE 4. Risk adjusted graft survival. A, Germany adjusted for all separate risk factors. B, Non-Germany adjusted for all separate risk factors. C, All transplantations adjusted for all separate risk factors.

be inferior is, however, currently still unclear. It could be related to differences in composition and viscosity ${ }^{2}$ which might lead to different effects in liver cell volume, efficiency of washout or to the presence of antioxidant agents. ${ }^{21,22}$ These effects would, in theory, especially affect short term graft survival.

The differences in donor, transplant and patient characteristics between HTK and UW are primarily a result of the national choice of preservation fluids. Germany, for example, used HTK in $97 \%$ of all procurements and in $93 \%$ of their transplantations (the difference is because of international exchange within Eurotransplant). When compared with all HTK transplantations in Eurotransplant, $75 \%$ of all HTKpreserved livers are transplanted in Germany. A country that has been struggling with one of the lowest DBD donor rates in Europe ${ }^{12}$ and has implemented a MELD based allocation system. Both are likely to impact posttransplantation outcome in a negative way (Figure 4). Because of the low donation rates, limits for liver allografts have been stretched and liver grafts are in general of lower quality; higher donor age, lab values and BMI. Also, because of the shortage of grafts, the waiting list expands and recipients will only be able to receive an offer when their MELD-score raises. ${ }^{23}$

For this reason, outcome was stratified for Germany versus all other countries. It is therefore interesting, that transplantations with HTK livers showed a trend for similar or better graft survival as compared with UW in both regions although this difference was not statistically significant. This statistical phenomenon where findings in subgroups are apparently contradictory to overall results is called a Simpson's paradox. It can exist when different sample sizes are compared of groups with different outcome. In this case, because of discrepancies in the use of preservation fluids between countries with different posttransplantation outcome. The latter affects outcome of UW livers in Germany: Germany almost exclusively uses HTK so livers perfused with UW are likely to originate from other ET countries. This is the case for livers that were not accepted for transplantation in the donor country.

The significant differences in outcome within Eurotransplant are also observed when results from ET are compared with the United States. The presented 1-year graft survival rates in non-German countries of about $83 \%$ are significantly lower than the approximately $90 \% 1$-year graft survival for first liver transplantations in the United States in $2016 .{ }^{24}$ We believe that a difference in liver quality between ET and the US attributes to this difference in outcome. This difference in donor quality was shown by Blok et al in $2012^{25}$ and is evident for donor age; about $66 \%$ of all livers used for a transplant in the United States in 2016 were from donors younger than 50 years old ${ }^{24}$ as compared with $36 \%$ in ET (median age, 55 years). ${ }^{23}$ This might be a result of regulation on center outcome as is done in the United States or by an assumed higher shortage of organs in ET. Regardless of the reason(s), the difference in donor quality shows that centers in ET have expanded their criteria for acceptable donors to increase the number of patients that can be transplanted and to decrease waiting list mortality. This strategy, however, comes at the cost of slightly inferior posttransplantation outcome.

In deciding what preservation fluid to use, the experience of surgeon and center should be the most important consideration. Our results indicate that no significant difference exists between both preservation fluids. Other aspects, like the lower viscosity, which is often appreciated by clinicians and the lower costs associated with the use of HTK might then also be taken into account.

\section{CONCLUSIONS}

The use of preservation fluids differs significantly per country within the Eurotransplant region. Histidinetryptophan-ketoglutarate is being used in the majority 
of liver transplantations and its use is increasing, in contrast to the use of UW. This retrospective database analysis shows that differences in outcome by preservation fluids are caused by regional differences in donor, recipient and transplant characteristics. These differences, rather than the used preservation fluid, cause the difference in outcome. When adjusted for these risk factors, no differences in graft survival exist between transplantations performed with livers that are preserved with either HTK or UW.

\section{ACKNOWLEDGMENTS}

The authors thank the Eurotransplant Liver and Intestine Advisory Committee and Eurotransplant for their support in providing the data. The authors acknowledge the effort of all liver transplant centers for providing data to the Eurotransplant registry.

\section{REFERENCES}

1. Eurotransplant. The donor. Chapter 9 of Eurotransplant manual. https:// www.eurotransplant.org/cms/index.php?page=et_manual. Published January 30, 2017. Accessed October 24, 2017.

2. Erhard J, Lange R, Scherer R, et al. Comparison of histidine-tryptophanketoglutarate (HTK) solution versus University of Wisconsin (UW) solution for organ preservation in human liver transplantation. A prospective, randomized study. Transpl Int. 1994;7:177-181.

3. Meine MH, Zanotelli ML, Neumann J, et al. Randomized clinical assay for hepatic grafts preservation with University of Wisconsin or histidinetryptophan-ketoglutarate solutions in liver transplantation. Transplant Proc. 2006;38:1872-1875.

4. Mangus RS, Tector AJ, Agarwal A, et al. Comparison of histidinetryptophan-ketoglutarate solution (HTK) and University of Wisconsin solution (UW) in adult liver transplantation. Liver Transpl. 2006;12:226-230.

5. Avolio AW, Agnes S, Nure E, et al. Comparative evaluation of two perfusion solutions for liver preservation and transplantation. Transplant Proc. 2006;38:1066-1067.

6. Mangus RS, Fridell JA, Vianna RM, et al. Comparison of histidinetryptophan-ketoglutarate solution and University of Wisconsin solution in extended criteria liver donors Richard. Liver Transplant. 2008;14:365-373.

7. Rayya F, Harms J, Martin AP, et al. Comparison of histidine-tryptophanketoglutarate solution and University of Wisconsin solution in adult liver transplantation. Transplant Proc. 2008;40:891-894.

8. Adam R, Delvart V, Karam V, et al. Compared efficacy of preservation solutions in liver transplantation : a long-term graft outcome study from the European Liver Transplant Registry. Am J Transplant. 2015;15:395-406.

9. Kaltenborn A, Gwiasda J, Amelung V, et al. Comparable outcome of liver transplantation with histidine-tryptophan-ketoglutarate vs. University of
Wisconsin preservation solution: a retrospective observational doublecenter trial. BMC Gastroenterol. 2014;14:169.

10. Stewart ZA, Cameron AM, Singer AL, et al. Histidine-tryptophanketoglutarate $(\mathrm{HTK})$ is associated with reduced graft survival in deceased donor livers, especially those donated after cardiac death. Am J Transplant. 2009;9:286-293.

11. Nashan B, Spetzler $V$, Schemmer P, et al. Regarding "compared efficacy of preservation solutions in liver transplantation: a long-term graft outcome study from the European Liver Transplant Registry". Am J Transplant. 2015;15:3272-3273. doi:10.1111/ajt.13513.

12. European Directorate for the Quality of Medicine (EDQM). Newsletter Transplant. https://www.edqm.eu/sites/default/files/newsletter_transplant_ volume_21_september_2016.pdf. Published 2016.

13. Adam R, Cailliez V, Karam V. Evaluation of HTK preservation solutions in liver transplantation: a long-term propensity-based analysis of outcome from the European liver transplant registry. Am J Transplant. 2017;17: 585-586.

14. DSO. Organ donation and transplantation in Germany annual report. https://www.google.com/url?sa=t\&rct=j\&q=\&esrc=s\&source=web\&cd= 1\&ved=0ahUKEwjGiMXE7b_bAhUE_4MKHWiqC8MQFgguMAA\&url= https\%3A\%2F\%2Fwww.dso.de\%2Fuploads\%2Ftx_dsodl\%2FDSO_ JB_D_2012_e.pdf\&usg=AOvVaw1vw_EWlpmrORbEAqwGL_ww. Published 2012.

15. Braat AE, Blok JJ, Putter $\mathrm{H}$, et al. The Eurotransplant donor risk index in liver transplantation: ET-DRI. Am J Transplant. 2012;12:2789-2796.

16. Blok JJ, Putter H, Rogiers X, et al. Combined effect of donor and recipient risk on outcome after liver transplantation: research of the Eurotransplant database. Liver Transpl. 2015;21:1486-1493.

17. Blok JJ, Ringers J, Putter H, et al. The combination of ET-DRI and recipient risk factors is predictive of graft failure after liver transplantation within the Eurotransplant region. Transpl Immunol. 2014;31:257.

18. Feng S, Goodrich NP, Bragg-Gresham JL, et al. Characteristics associated with liver graft failure: the concept of a donor risk index. Am J Transplant. 2006;6:783-790.

19. Dutkowski P, Oberkofler CE, Slankamenac K, et al. Are there better guidelines for allocation in liver transplantation? Ann Surg. 2011;254: 745-754.

20. Feng L, Zhao N, Yao X, et al. Histidine-tryptophan-ketoglutarate solution vs. University of Wisconsin solution for liver transplantation: a systematic review. Liver Transpl. 2007;13:1125-1136.

21. Lee CY, Mangino MJ. Preservation methods for kidney and liver. Organogenesis. 2009;5:105-112.

22. Feng $X N, X u X$, Zheng SS. Current status and perspective of liver preservation solutions. Hepatobiliary Pancreat Dis Int. 2006;5:490-494.

23. Eurotransplant International Foundation. Annual Report 2016. Eurotransplant International Foundation. www.eurotransplant.org. Published 2016.

24. OPTN. National Data. https://optn.transplant.hrsa.gov/data/view-datareports/national-data/. Published 2018. Accessed April 11, 2018.

25. Blok JJ, Braat AE, Adam R, et al. Validation of the donor risk index in orthotopic liver transplantation within the Eurotransplant region. Liver Transpl. 2012;18:112-119. 